\title{
PLASMA WAKEFIELD ACCELERATION OF AN INTENSE POSITRON BEAM: CORRELATION BETWEEN TIME-RESOLVED AND TIME- INTEGRATED ENERGY DIAGNOSTICS
}

\author{
B.E. Blue ${ }^{* \dagger}{ }^{\dagger}$ P. Muggli, ${ }^{\S}$ M.J. Hogan,${ }^{\ddagger}$ C.L. O’Connell, ${ }^{\ddagger}$ C. Clayton, ${ }^{*}$ F.-J. Decker ${ }^{\ddagger}$ C. Huang, ${ }^{*}$ R. \\ Iverson, ${ }^{\ddagger}$ K.A. Marsh, ${ }^{*}$ W.B. Mori, ${ }^{*}$ D. Walz, ${ }^{\ddagger}$ T.C. Katsouleas, ${ }^{\S}$ R. Siemann, ${ }^{\ddagger}$ C. Joshi ${ }^{*}$ \\ *University of California Los Angeles, Los Angeles, CA 90095 \\ ${ }^{\S}$ University of Southern California, Los Angeles, CA 90089 \\ ${ }^{*}$ Stanford Linear Accelerator Center, Menlo Park, CA 94025
}

\begin{abstract}
The E162 experiment at the Stanford Linear Accelerator Center was the first experiment in which a positron beam gained energy in a plasma wakefield accelerator [1]. A single positron bunch both excited (gave energy to) and witnessed (extracted energy from) the plasma wakefield. The energy dynamics within the single positron bunch were measured in a dispersive section of the beamline with both time-resolved (1 ps streak camera) and time-integrated (CCD camera) diagnostics. This paper will correlate the energy gain and loss measurements from both diagnostics.
\end{abstract}

\section{MOTIVATION}

The bunch length $\left(\sigma_{\mathrm{z}} / \mathrm{c}=2.4 \mathrm{ps}\right)$ used in the E162 experiment was long enough to be temporally resolved using current state of the art streak cameras. However, streak cameras do not exist to temporally resolve the dynamics within the beam as the bunch length is shortened in future experiments (E164: $\sigma_{\mathrm{z}} / \mathrm{c} \sim 300 \mathrm{fs}$; E164X: $\left.\sigma_{z} / \mathrm{c} \sim 30 \mathrm{fs}\right)$. Therefore, the use of timeintegrated diagnostics is mandated and a methodology must be generated in order to extract the accelerated particles from the back of the bunch from data that contains both the accelerated and decelerated particles in the entire bunch.

\section{E162 EXPERIMENT}

The experimental setup has been described in detail elsewhere [2], but the salient features will be briefly introduced. A $730 \mu \mathrm{m}$ long, $40 \mu \mathrm{m}$ round positron bunch containing $1.2 \times 10^{10}$ particles was propagated through a 1.4 meter long plasma of density $0-2 \times 10^{14} \mathrm{~cm}^{-3}$. Upon exiting the plasma, the beam was imaged onto a $1 \mathrm{~mm}$ thick aerogel Cherenkov radiator using a magnetic imaging spectrometer with $291 \mathrm{MeV} / \mathrm{mm}$ of dispersion in the vertical plane. The visible Cherenkov radiation was imaged onto the slit of a streak camera and onto a CCD camera. By measuring the change in position of individual beam slices in the dispersive plane with the streak camera, the energy distribution in a single bunch could be measured. This paper will address how the

\footnotetext{
† Present Address: Lawrence Livermore National Laboratory Livermore, CA 94550

\# Email: blue3@1lnl.gov
}

energy gain in the back of the positron bunch and energy loss by the core of the bunch can be measured on the CCD camera at the expense of losing temporal information.

\section{TEMPORALLY RESOLVED ENERGY CHANGE OF A SINGLE POSITRON BUNCH}

As a ultrarelativistic positron beam propagates through an underdense plasma, the highly mobile plasma electrons are pulled in towards the beam by the beam's space charge field. As these plasma electrons from various radii arrive on the axis of the beam at various times and overshoot, they create a wakefield structure that has complex longitudinal and transverse electric field components [3]. The energy change induced on a positron bunch by the plasma wakefield is shown in Figure 1. The main body of the beam loses $52 \pm 16 \mathrm{MeV}$ driving the plasma wave, while the back of the beam extracts energy from the wave and in turn gains $79 \pm 19$ $\mathrm{MeV}$.

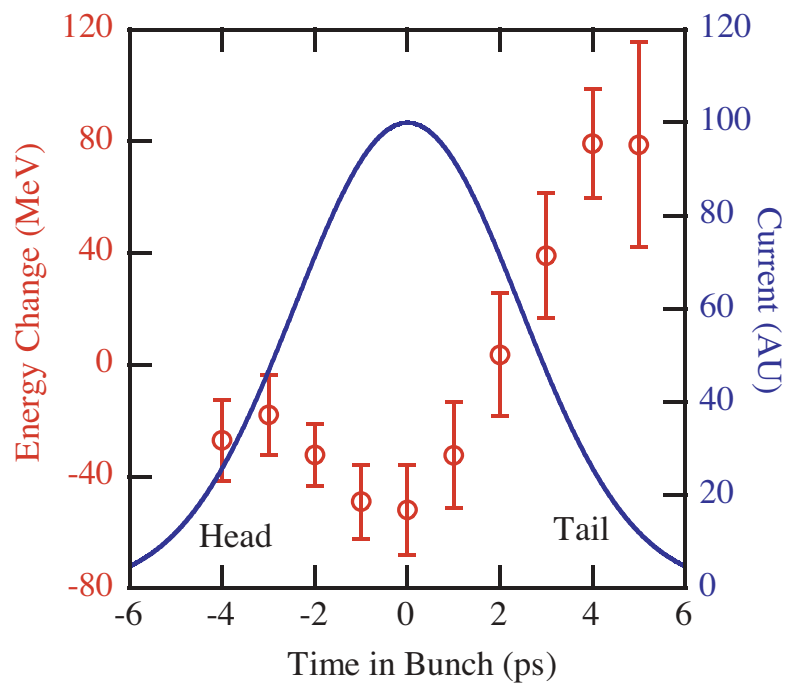

Figure 1: Time slice analysis (red circles) of the energy dynamics imparted on a single positron bunch (blue line) after it passed through $1.4 \mathrm{~m}$ of $1.8 \times 10^{14} \mathrm{~cm}^{-3}$ plasma. The front half of the beam losses energy driving the plasma wave, while the back of the beam is accelerated by the plasma wave. 


\section{METHOD OF MEASURING ENERGY GAIN FROM A TIME-INTEGRATED CCD DIAGNOSTIC}

The time-resolved streak camera diagnostic allowed us to measure the energy of $1 \mathrm{ps}$ slices within a single positron bunch. Furthermore, the energy of each slice could be measured independently from the energy and/or spot size fluctuations of other beam slices. However, when a time integrated diagnostic is used, the energy dispersed profile will contain the superimposed contributions of spot size (focusing and defocusing) and energy gain/loss of each beam slice. In an ideal design, the dispersion would be much greater than the undispersed spot size and therefore spot size fluctuations could be neglected. Due to hardware constraints in our experiment, the spot size was on the order of the dispersion. This implies that the measured energy gain will consist of a shift in the distribution of particles whose contribution to the overall energy dispersed profile will be partially masked by other particles which were not accelerated.

With a general overview of measuring the energy gain on a time-integrated diagnostic now introduced, the specific method used in our measurement will now be introduced. The first step was in determining the extent of the focusing/defocusing of the positron beam. Although the complex wakefields setup non-uniform focusing and defocusing along the length of the bunch [4], the overall extent of the focusing for the entire bunch can be measured. This is shown in Figure 2. The plasma off RMS spot size at the Cherenkov radiator plane is about $210 \mu \mathrm{m}$, while at the density of interest for our analysis $\left(1.8 \times 10^{14} \mathrm{~cm}^{-3}\right)$, the spot size has blown up by $\sim 10 \%$ to $230 \mu \mathrm{m}$. In energy space this width corresponds to an energy width of $\sim 70 \mathrm{MeV}$.

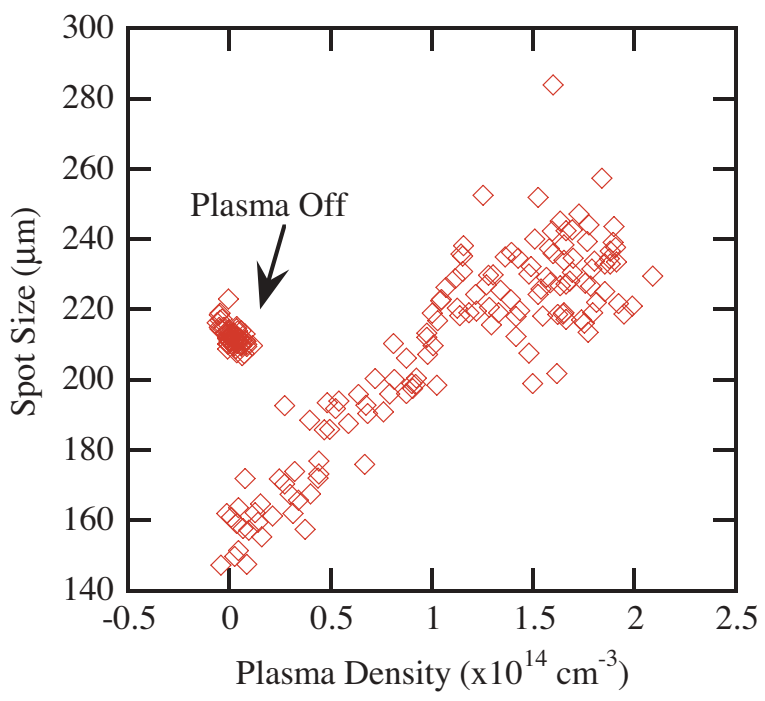

Figure 2: Plasma focusing of the positron beam.

The next step is to look at the profiles of the beam in the energy dispersed plane. These profiles were generated by acquiring a time-integrated CCD image of the positron beam at the Cherenkov plane. The image was integrated in the non-dispersive plane to remove ambiguity about which part of the beam was sampled by the profile. Figure 3 shows the acquired profiles for both the plasma off (dashed blue line) and plasma on (solid red line) cases. The peak of the plasma off case was normalized to 28.5 $\mathrm{GeV}$ since we are interested in changes to the beam energy. Since the contributions to the profiles are from both spot size and energy change, the $\mathrm{x}$-axis is labeled with both units. We know that an energy change in the beam is distorting the profile because the profile is not symmetric about the origin, a fact confirmed from the time-resolved streak camera data. Focusing and defocusing will symmetrically expand or contract the distribution, while a change in energy will shift the distribution towards the left (energy loss) or right (energy gain). As shown in Figure 3, at a density of $1.8 \times 10^{14}$ $\mathrm{cm}^{-3}$, the beam losses energy driving the plasma wave, and additionally, part of the beam has gained energy.

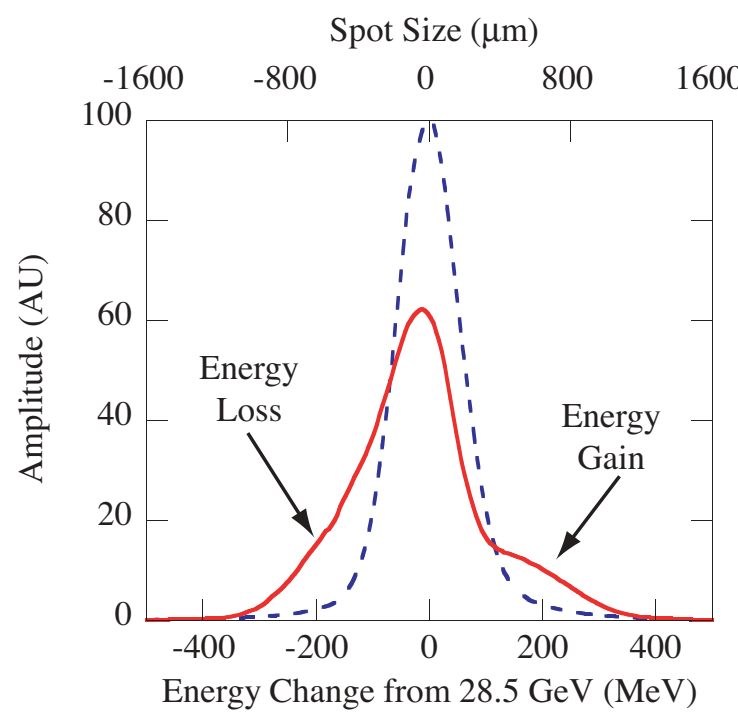

Figure 3: Profile of positron beam in the dispersive plane. The profile of the beam sans plasma (dashed blue line) is due to the spot size of the beam. The profile of the beam after propagating through $1.4 \mathrm{~m}$ of plasma (solid red line) includes the effect of focusing/defocusing and energy gain/loss.

To extract the magnitude of the energy gain, a Gaussian was fit to the energy gain distribution of Figure 3. This fit is shown as a dashed black line in Figure 4. Since the dispersion induced shift in position is on the order of the beam RMS spot size, part of the Gaussian fit will be masked by the rest of the beam. This is shown in Figure 4 as the dashed black line shown inside of the beam profile (solid red line). The measured energy gain of $90 \mathrm{MeV}$ was determined from the mean position of the Gaussian fit. This is in reasonable agreement with the streak camera data which measured an average energy gain of 79 $\mathrm{MeV}$ over the last two slices of the beam as seen in Fig. 1. 


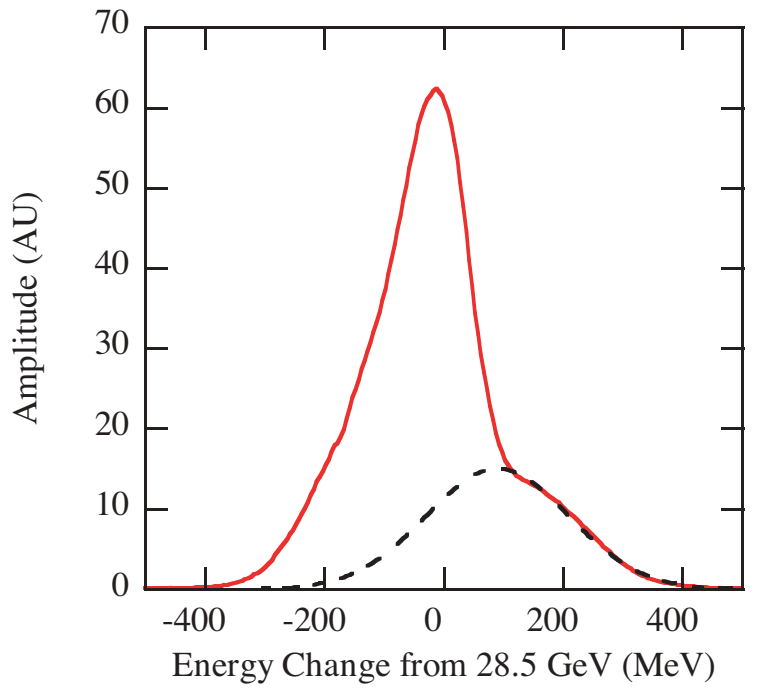

Figure 4: Profile of the plasma modulated positron beam in the dispersive plane (solid red line) and a Gaussian fit to energy gain (dashed black line) in the back of the positron bunch. An energy gain of $90 \mathrm{MeV}$ was measured.

To determine the average energy loss of the beam, a center-of-energy (similar to center-of-mass) calculation was made on the energy dispersed profile. To remove the energy gain contribution to the profile, the Gaussian fit to the energy gain was subtracted from the profile. The resultant "energy-loss" profile is shown as a dashed black line in Fig. 5. The center-of-energy calculation resulted in an average energy loss by the bulk of the beam of 63 $\mathrm{MeV}$. This is in reasonable agreement with the timeresolved streak camera data that measured an energy loss by the centroid of the beam ( 0 ps slice) of $52 \pm 16 \mathrm{MeV}$.

We are currently attempting more sophisticated fitting routines to cases where the beam size in the dispersion plane is dominated by initial energy spread, not focusing or defocusing effects. This will be extremely important for the ultra-short beam cases of E164 $\left(\sigma_{z} \sim 100 \mu \mathrm{m}\right)$ and E164X $\left(\sigma_{z} \sim 10 \mu \mathrm{m}\right)$ where no comparison with a timeresolved diagnostic will be possible.

\section{CONCLUSIONS}

We have used two different methods to measure the energy gain and loss induced on a positron beam in the plasma wakefield accelerator. The time-resolved diagnostic measured an energy gain of $79 \pm 19 \mathrm{MeV}$ and the time-integrated diagnostic measured an energy gain of $90 \mathrm{MeV}$. The time-resolved diagnostic measured an energy loss of $52 \pm 16 \mathrm{MeV}$ of the beam centroid, while the time-integrated diagnostic measured an average energy loss of $63 \mathrm{MeV}$. These two diagnostic measurements are in reasonable agreement with each other, therefore future plasma wakefield acceleration experiments will be able to use time-integrated diagnostics in order to measure energy loss and gain.

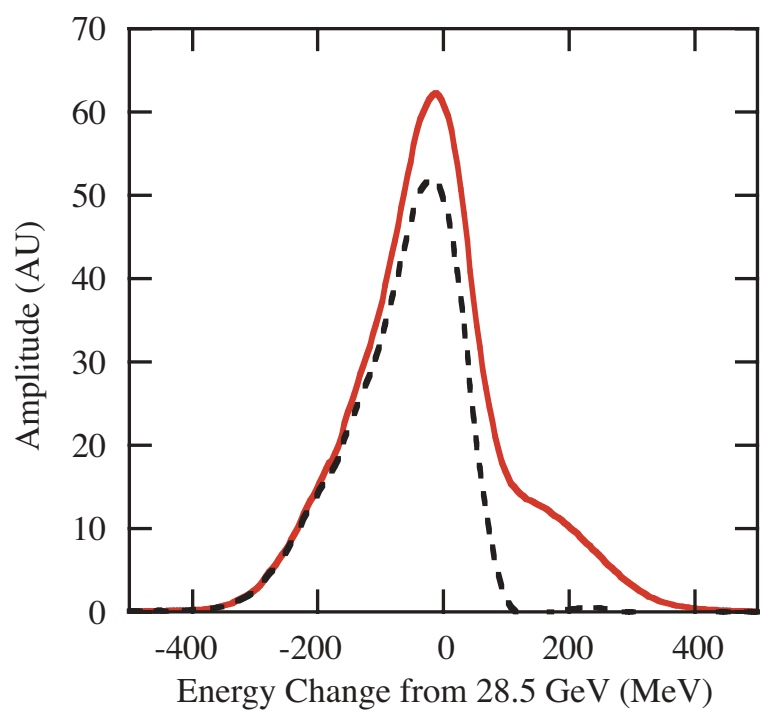

Figure 5: Profile of the plasma modulated positron beam in the dispersive plane (solid red line) and the distribution of charge with the contribution of energy gain subtracted (dashed black line). An average energy loss by the bulk of the beam of $63 \mathrm{MeV}$ was measured.

\section{ACKNOWLEDGEMENTS}

This work is supported by U.S. DoE grant numbers DEFG03-92ER40745, DE-AC03-76SF00515, DE-FG0398DP00211, and DE-FG03-92ER40727 and by NSF grant numbers ECS-9632735, DMS-9722121, and PHY00787157.

\section{REFERENCES}

[1] B. Blue et al., Accepted for Publication in Physical Review Letters.

[2] C. Joshi et al., Phys. Plasmas 9, 1845 (2002).

[3] S. Lee et al., Phys. Rev. E 64, 045501 (2001).

[4] M.J. Hogan et al., Accepted for Publication in Physical Review Letters. 